\section{Muscle and Movement Representations in the Primary Motor Cortex}

\author{
Shinji Kakei, ${ }^{1}$ Donna S. Hoffman, ${ }^{1,3}$ Peter L. Strick ${ }^{1,2,3 *}$
}

What aspects of movement are represented in the primary motor cortex (M1): relatively low-level parameters like muscle force, or more abstract parameters like handpath? To examine this issue, the activity of neurons in $M 1$ was recorded in a monkey trained to perform a task that dissociates three major variables of wrist movement: muscle activity, direction of movement at the wrist joint, and direction of movement in space. A substantial group of neurons in M1 (28 out of 88) displayed changes in activity that were muscle-like. Unexpectedly, an even larger group of neurons in M1 (44 out of 88) displayed changes in activity that were related to the direction of wrist movement in space independent of the pattern of muscle activity that generated the movement. Thus, both "muscles" and "movements" appear to be strongly represented in M1.
There has been long-standing controversy over whether "muscles" or "movements" are represented in the primary motor cortex (M1) (1). From a contemporary perspective, this question can be recast: What aspects of movement are encoded in the activity of M1 neurons: relatively low-level movement parameters like muscle force, or more abstract movement parameters like handpath? Since the pioneering work of Evarts (2), this question has been examined by recording the activity of single neurons in awake trained primates [for example, (3-10)]. Early experiments examined M1 activity in relation to simple finger and wrist movements (3). The discharge of many M1 neurons in these studies covaried with movement parameters such as static and dynamic force. These results led to the view that M1 is concerned with the generation of movement in terms of an "intrinsic" parameter space related to one or more of a number of variables including aspects of joint kinematics, joint torques, and the detailed pattern of muscle activity at a single joint.

A different perspective has come from studies of M1 activity during reaching movements (4). In some experiments, the activity of M1 neurons, as a population, covaried with the trajectory of hand movement and signaled its instantaneous movement direction and velocity. These and similar results led to the view that M1 is concerned with the generation of movement in terms of an "extrinsic"

${ }^{1}$ Research Service (151S), Veterans Affairs Medical Center; ${ }^{2}$ Department of Neurosurgery and ${ }^{3}$ Department of Neuroscience and Physiology, State University of New York Health Science Center at Syracuse, Syracuse, NY 13210, USA.

*To whom correspondence should be addressed at VA Medical Center, Research Service (151S), 800 Irving Avenue, Syracuse, NY 13210, USA. E-mail: strickp @hscsyr.edu parameter space related to the motion of the hand, the location of the target in space, or both $(4,9,10)$. However, the results of other experiments of M1 activity during reaching movements made under altered load conditions or with different arm postures produced evidence for coding in an intrinsic parameter space (5).

To address this controversy, we developed a paradigm that dissociates three different coordinate frames related to wrist movements: extrinsic (related to the direction of movement in space), muscle (related to the activity of individual or groups of muscles), and joint (related to the angle of the wrist joint) (11-13). Our paradigm takes advantage of two features of the wrist joint. First, the wrist rotates along two axes: flexion-extension and radial-ulnar deviation. Second, the direction of wrist movement in space depends on the posture of the forearm. For example, an upward movement in space is produced by wrist flexion when the forearm is held in supination, or by wrist extension when the forearm is held in pronation. We trained a monkey to perform step-tracking movements of the right wrist that required eight different combinations of wrist flexion-extension and radial-ulnar deviation (Fig. 1B). These movements were performed while the forearm was
Fig. 1. Dissociation of muscle space from extrinsic and joint space. (A) Schematic of monkey right hand gripping the handle of the manipulandum in three wrist postures. (Left) Pro position, fully pronated; (right) Sup position, fully supinated; (center) Mid position, midway between the two. (B) Average movement trajectories to eight peripheral targets in three wrist postures. The target locations required $20^{\circ}$ changes in the angle of the wrist joint. Each trace is the average of 10 movements to the same target location. The dotted lines indicate the straight line trajectory to each target. Rad: radial deviation; Ext: extension; Uln: ulnar deviation; Flx: flexion. (C) Distribution of preferred directions (PDs) of three wrist muscles when the limb was in the three wrist postures (three to four recordings from each muscle). ECRB: extensor
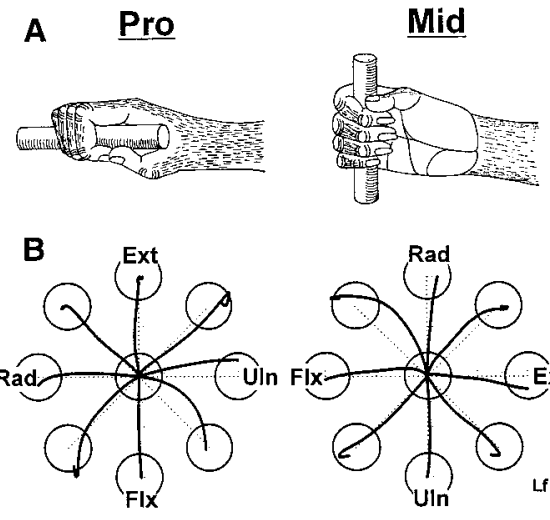

C
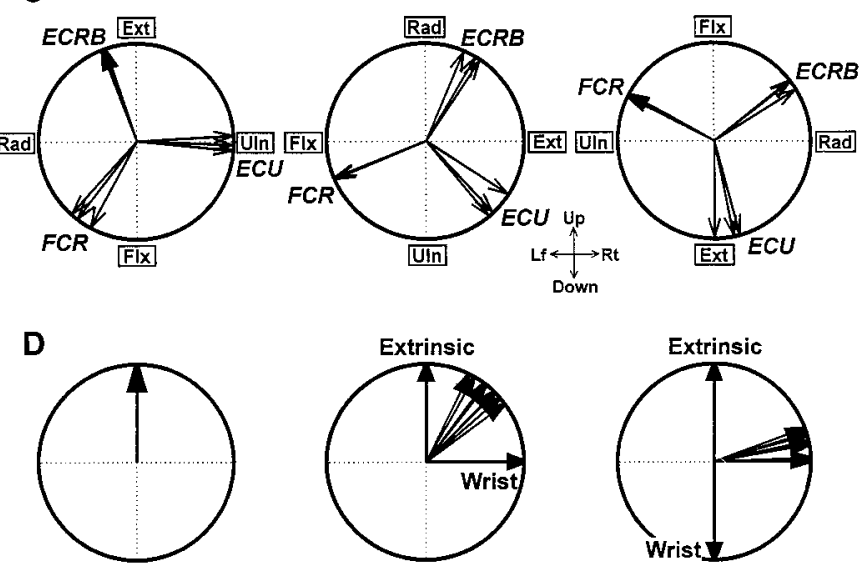

A Pro
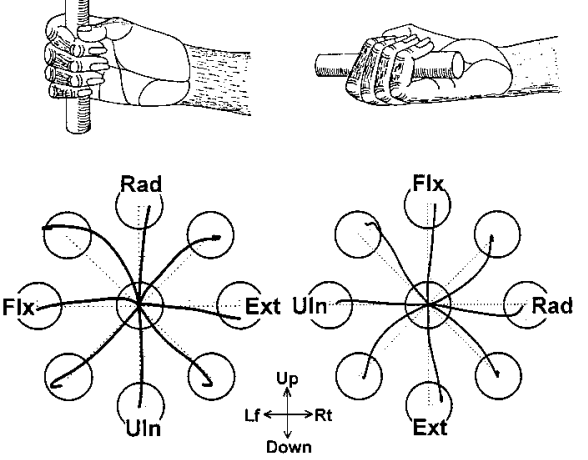

carpi radialis brevis;

ECU: extensor carpi ulnaris; FCR: flexor carpi radialis. (D) Normalized shifts of PDs with wrist rotations from Pro to Mid and from Pro to Sup. PDs in the Pro position are set to $0^{\circ}$ (represented as a single vector in the left circle). Each unlabeled vector in the middle and right circles indicates the relative shift of a PD of the same three muscles from Pro to Mid and from Pro to Sup, respectively. Vectors labeled "Extrinsic" represent the ideal PDs of vectors fixed to an extrinsic coordinate frame. Vectors labeled "Wrist" represent ideal PDs of vectors fixed to the wrist joint. 
held in each of three different postures: pronated (Pro), supinated (Sup), and midway between the two (Mid) (Fig. 1A).

Once the animal was trained, we examined the patterns of muscle activity associated with performance of the task. We recorded the activity of 27 muscles in the forearm, upper arm, and shoulder (14-16). Only four wrist muscles and three finger muscles (17) were active before movement onset in all three postures. These muscles displayed phasic agonist bursts whose amplitudes varied for different directions of movement. The variation in amplitude for each muscle was well fit by a cosine function (18). We determined the preferred di- rection (PD) for the seven task-related muscles while the limb was in each of the three separate postures (Fig. 1C). When the forearm was rotated clockwise from Pro to Mid, and then from Mid to Sup, the PDs of all seven muscles rotated clockwise as well. The shift in the PD for individual muscles was always less than $90^{\circ}$ for the full $180^{\circ}$ of forearm rotation (Fig. 1D) (mean \pm $\mathrm{SD}=71.1^{\circ} \pm 9.8^{\circ}$, range $=46^{\circ}$ to $90^{\circ}$ ) (19). This result indicates that the coordinate frame associated with muscles can be distinguished from other coordinate frames because it rotates less than the coordinate frame related to the wrist joint $\left(180^{\circ}\right)$, but more than an extrinsic coordinate frame
Fig. 2. Directional tuning of three single neurons recorded in the primary motor cortex (M1). Each column shows the activity for one of the three wrist postures. Each row shows a neuron's activity for the same direction of movement in "extrinsic" space. Each line in a raster represents a single trial. The single letters in the upper left-hand corner of each raster indicate the direction of "joint" movement. All rasters are aligned on movement onset (indicated by small filled triangles at the bottom center of each raster). $R$ : radial deviation; E: extension; $\mathrm{U}$ : ulnar deviation; F: flexion. The units are in milliseconds. (A) Muscle-like neuron in M1. This neuron showed a clear shift in PD $\left(79^{\circ}\right)$ when the wrist was rotated from Pro to Sup. The size of this shift in PD is quite similar to that of muscles (Figs. $1 D$ and 4B). We estimated that this neuron
$A$

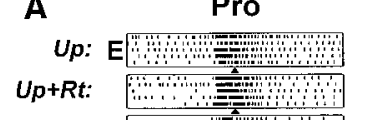

Rt: U

Dn+Rt:

Dn: F

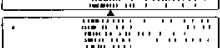

$D n+L f$ :

Lf:
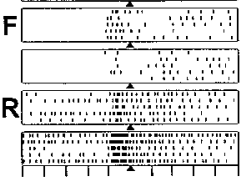

B
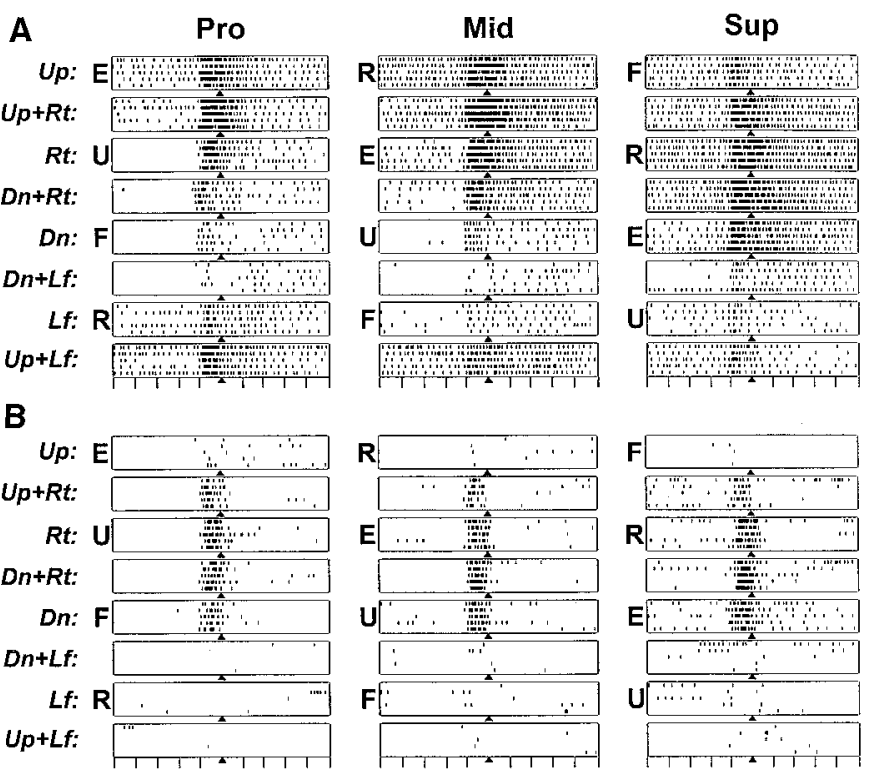

Up+Rt:

Rt: U
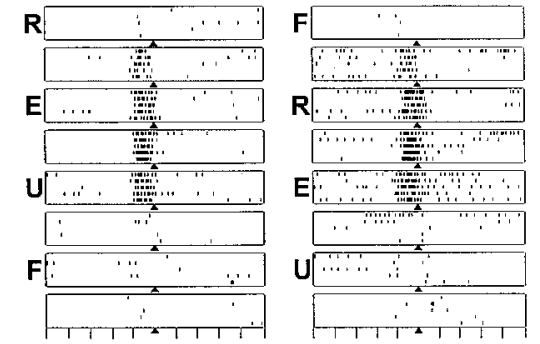

C Up: $E$ a

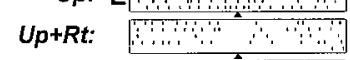

Rt: U[?

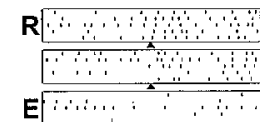

$D n+R t$
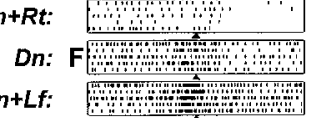

Dn+Lf: $\quad$ How

Lf: $R$ R

Up+Lf:

Lf:
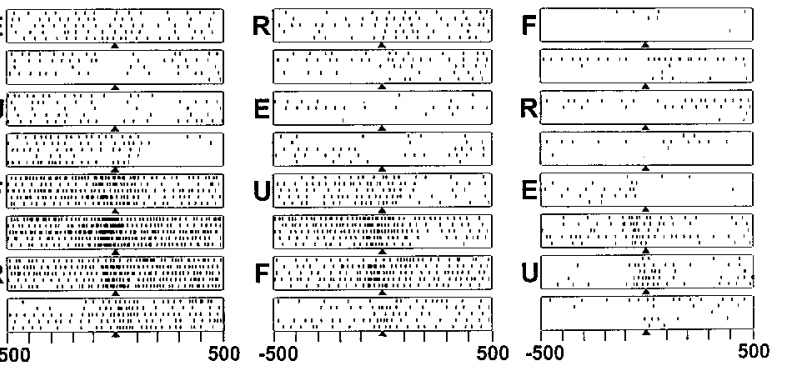

was recorded in the

rostral bank of the central sulcus, $\sim 4 \mathrm{~mm}$ from the cortical surface. Intracortical stimulation at the recording site evoked contractions of ECRB at a threshold of $10 \mu \mathrm{A}$. The neuron was activated by passive flexion and ulnar deviation of the wrist and thumb adduction. (B) Extrinsic-like neuron in M1. This neuron displayed a burst of activity before movement onset for the same target locations, irrespective of the wrist posture. The activity of this neuron showed no shift in PD with wrist rotation. This neuron's response appears to be best related to an extrinsic coordinate frame (Fig. 1D). We estimated that this neuron was recorded in the rostral bank of central sulcus, $\sim 6 \mathrm{~mm}$ from the cortical surface. Intracortical stimulation at the recording site evoked contractions of EDC at a threshold of $2 \mu \mathrm{A}$. The neuron was activated by passive extension of digits 1 to 5 . The PD of EDC shifted in a manner similar to that of ECRB (Fig. 1C), but was rotated clockwise $25^{\circ}$ to $37^{\circ}$ in relation to the PD of ECRB. (C) Extrinsic-like neuron with activity strongly influenced by joint posture. The activity of this neuron showed no shift in PD with wrist rotation. However, both the peak activity and background discharge rate displayed a systematic modulation with a change in wrist posture. We estimated that this neuron was recorded in the rostral bank of the central sulcus, $\sim 4 \mathrm{~mm}$ from the cortical surface. Intracortical stimulation at the recording site evoked wrist extension and ulnar deviation of digits 4 and 5 at a threshold of $10 \mu \mathrm{A}$. The neuron was activated by passive extension and ulnar deviation of the wrist. related to the direction of movement in space $\left(0^{\circ}\right)$.

On the basis of these observations, we reasoned that neurons signaling information in a muscle- or jointlike coordinate frame will have PDs that shift $46^{\circ}$ to $90^{\circ}$ (muscle) or $180^{\circ}$ (joint) when wrist movements are made with the forelimb in different postures. On the other hand, neurons signaling information in an extrinsic-like coordinate frame will have PDs that are relatively stable under the same task conditions. Therefore, to determine the coordinate frame for M1 neurons, we recorded the activity of single neurons in M1 while a monkey performed our task (20-22). We found 125 neurons in the hand area of M1 that displayed movement-related activity. Of these, 88 had directionally tuned (23) activity in all three wrist postures. These neurons are the subject of this report.

We calculated the PD of each directionally tuned neuron for the three separate postures $(18,24)$. This allowed us to determine the shift in PD for the $180^{\circ}$ clockwise rotation from the Pro to Mid to Sup postures. On the basis of this analysis, the 88 directionally tuned neurons formed three groups. One group ( $n=28 / 88)$ showed orderly and relatively large $\left(>40^{\circ}\right)$ shifts in PD (mean \pm $\mathrm{SD}=70.2^{\circ} \pm 21.9^{\circ}$, range $=+43^{\circ}$ to $\left.+147^{\circ}\right)$ (Figs. 2A and 3A). The shifts for all but one of the neurons in this group paralleled those of muscles (Fig. 4). We consider neurons with a shift in $\mathrm{PD}$ between $40^{\circ}$ and $110^{\circ}$ as having "muscle-like" properties. Thus, the activity of neurons in this group appears to represent movement in an intrinsic coordinate frame and may encode commands for single or groups of muscles.

In contrast, a second, larger group of neurons $(n=44 / 88)$ showed more modest $\left(<35^{\circ}\right)$ shifts in $\mathrm{PD}\left(\right.$ mean $\pm \mathrm{SD}=12.3^{\circ} \pm$ $12.5^{\circ}$, range $=-15.3^{\circ}$ to $\left.+32.8^{\circ}\right)$ (Figs. $2 \mathrm{~B}$ and $3 \mathrm{~B})$. In most instances $(n=38 / 44)$, the shifts in PD were not statistically significant $(P>0.2)$ (for example, Figs. $2 \mathrm{~B}$ and $3 \mathrm{~B}$ ). The absence of shifts for this group of neurons mirrored the stability of an extrinsic coordinate frame. We consider neurons with a limited shift in $\mathrm{PD}\left(-20^{\circ}\right.$ to $\left.+35^{\circ}\right)$ as having "extrinsic-like" properties. Thus, the activity of this group of neurons may encode commands for the direction of wrist movement in space, independent of the pattern of muscle activity that generates the movement.

A third, small group of neurons $(n=$ 16/88) showed unsystematic shifts in PD. For example, some neurons in this group displayed a clockwise shift in PD for a change in posture from Pro to Mid, but showed a counterclockwise shift in PD for a change from Mid to Sup. The neurons in this group will not be considered further.

A change in the posture of the forelimb alters the muscle and joint mechanics for 
movements at the wrist joint. As a consequence, one would expect the amount of muscle activity necessary to produce a given change in joint angle to vary in the different postures. Indeed, changes in limb posture strongly modulated $(\Delta>30 \%)$ the movement-related activity of most (17/23 recordings) forearm muscles. Similarly, changes in posture also modulated the movement-related activity of most muscle-like (18/27) and extrinsic-like (27/44) neurons in M1 (for example, Figs. 2C and 3C). On the other hand, a sizable number of extrinsic-like neurons in M1 (17/44) did not display this type of modulation. The absence of modulation by posture is another property that distinguishes these extrinsic-like neurons from the motor apparatus in the periphery and its associated intrinsic coordinate frame.

Extrinsic- and muscle-like neurons were recorded at similar locations and depths within M1. Intracortical stimulation at the recording sites of both groups of neurons evoked contractions of wrist or finger muscles (or both) in the forearm at comparable thresholds (25). The changes in neuron activity for extrinsic- and muscle-like neurons occurred at approximately the same time in relation to movement onset (25). The similarity in these properties suggests that the two groups of neurons may be at comparable levels of intracortical processing within M1. In other words, at present we have no means to determine whether either or both groups of neurons is a source of descending commands to the spinal cord.

Our results help to resolve the controversy concerning parameter representation in M1 (1). We found that M1 contains at least two distinct groups of neurons. A substantial portion of our sample displayed properties that were muscle-like [see also $(2,3,5)]$. On the other hand, an even larger portion of our sample was related to a more abstract movement parameter, direction of action $(4,9,10)$. Thus, the answer to the question of whether muscles (an intrinsic parameter) or movements (an extrinsic parameter) are represented in M1 is that, at the single-neuron level, both are strongly represented.

Our observations may also provide insight into the type of sensorimotor transformations that take place in M1. Some have modeled the process for generating goaldirected movements as a series of coordinate transformations beginning with the representation of the target in external
Fig. 3. Spatiotemporal maps of activity of the same M1 neurons illustrated in Fig. 2. Neurons $(\mathrm{A}),(\mathrm{B})$, and $(\mathrm{C})$ in this figure correspond to neurons $A, B$, and $C$ in Fig. 2. To construct these maps, we calculated averaged spike numbers in a 50-ms time window, sliding the time window by $25 \mathrm{~ms}$, from -500 to $+500 \mathrm{~ms}$ relative to the movement onset. The calculation was performed for each movement direction in each wrist posture. Then, contour plots of the spatiotemporal distribution of the neuron activity were generated with Surfer (Golden Software, Golden, Colorado). The maximum activity for any of the three wrist postures in the $50-\mathrm{ms}$ analysis window was normalized to $100 \%$ (A: 155 ; B: 98; C: 124 spikes per second). PDs in the Pro position were set to $0^{\circ}$ in order to demonstrate the amount of the shift. PDs of neuron activity (18) for each posture are indicated by arrows.

Movement onsets are indicated by triangles. space and ending with the representation of muscle activation patterns in an intrinsic coordinate space $(6,26)$. For example, Alexander and Crutcher (6) have described this model as "a sequence of computations that successively determine 1) the location of the target in space, 2) the hand trajectory needed to acquire the target, 3) the joint kinematics needed to achieve the trajectory (inverse kinematics), 4) the joint torques needed to satisfy the kinematic constraints (inverse dynamics) and 5) the patterns of effector ('muscle') activation needed to satisfy the required dynamics." One interpretation of our results is that M1 is involved in multiple stages of this process, and not just the final computation [that is, defining patterns of muscle activity; see also (27)]. Indeed, interactions among extrinsic-like neurons unmodulated by joint posture, extrinsic-like neurons modulated by joint posture, and muscle-like neurons may be the neural correlate of transformations between different coordinate frames.

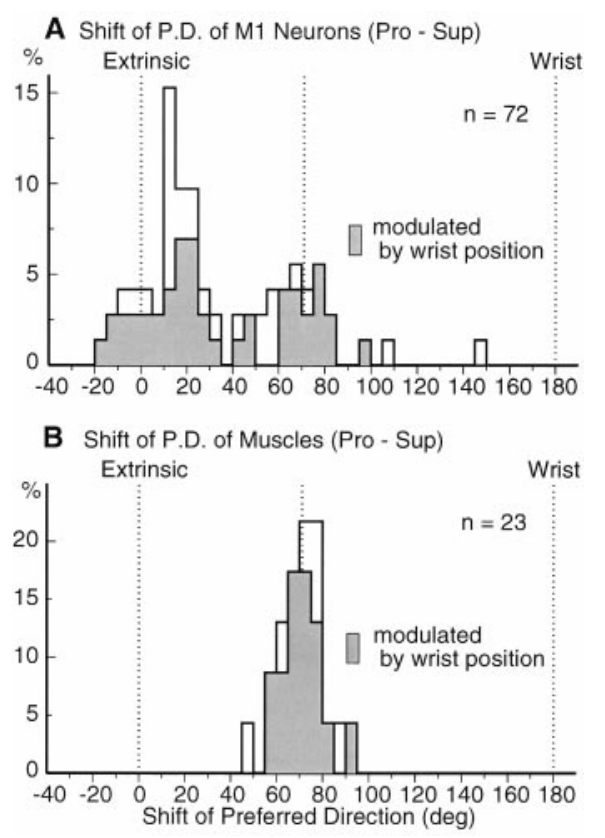

Fig. 4. Distribution of shifts of PDs from Pro to Sup. (A) Shift of PDs of M1 neurons. A clockwise shift is represented as positive. The vertical dotted line labeled "Extrinsic" indicates an ideal extrinsic-like vector that does not shift with changes in posture. The vertical line labeled "Wrist" indicates an ideal vector fixed to the wrist joint that shifts by $180^{\circ}$ with changes in wrist posture. The vertical line in the middle indicates the averaged shift $\left(71.1^{\circ}\right)$ of the seven forearm muscles that displayed early phasic EMG activity that was directionally tuned for all three postures. Shaded areas indicate neurons whose peak activity changed by more than $30 \%$ with wrist posture. (B) Shift of PDs of forearm muscles (23 recordings from seven forearm muscles). Shaded areas indicate EMG recordings whose peak activity changed by more than $30 \%$ with wrist posture. 


\section{R E P O R T S}

\section{References and Notes}

1. E. V. Evarts, in Neurophysiological Basis of Normal and Abnormal Motor Activities, M. D. Yahr and D. P. Purpura, Eds. (Raven, New York, 1967), pp. 215-253.

2. Neurophysiol. 31, 14 (1968).

3. W. T. Thach, ibid. 41, 654 (1978); A. M. Smith, M.-C. Hepp-Reymond, U. R. Wyss, Exp. Brain Res. 23, 315 (1975); E. E. Fetz and P. D. Cheney, J. Neurophysiol. 44, 751 (1980); P. D. Cheney and E. E. Fetz, ibid., P. 773; E. V. Evarts, C. Fromm, J. Kröller, V. A. Jennings, ibid. 49, 1199 (1983); E. J. Buys, R. N. Lemon, G. W. H. Mantel, R. B. Muir, J. Physiol. 381, 529 (1986).

4. A. P. Georgopoulos, J. F. Kalaska, R. Caminiti, J. T. Massey, J. Neurosci. 2, 1527 (1982); Exp. Brain Res. Suppl. 7 (1983); A. P. Georgopoulos, R. E. Kettner, A. B. Schwartz, J. Neurosci. 8, 2928 (1988).

5. J. F. Kalaska, D. A. D. Cohen, M. L. Hyde, M. Prud'homme, J. Neurosci. 9, 2080 (1989); S. H. Scott and J. F. Kalaska, J. Neurophysiol. 77, 826 (1997)

6. Reviewed in G. E. Alexander and M. D. Crutcher, J. Neurophysiol. 64, 133 (1990).

7. _ ibid., P. 164; L. Shen and G. E. Alexander ibid. 77, 1171 (1997).

8. R. Caminiti, P. B. Johnson, A. Urbano, J. Neurosci. 10, 2039 (1990).

9. A. P. Georgopoulos, J. Ashe, N. Smyrnis, M. Taira, Science 256, 1692 (1992)

10. A. B. Schwartz, J. Neurophysiol. 70, 28 (1993).

11. The monkey was trained to perform rapid step-tracking movements of the wrist for a liquid reward. The animal sat in a primate chair with its forearm supported and grasped the handle of a lightweight, lowfriction manipulandum [see Fig. 1 in D. S. Hoffman and P. L. Strick, J. Neurosci. 6, 3309 (1986)]. The device rotated along the two axes of wrist joint motion: flexion-extension and radial-ulnar deviation. Potentiometers at each axis measured rotation in the two planes of joint motion. The monkey faced a large computer screen that displayed a "cursor" and a "target." The cursor was a small, filled circle that moved in proportion to the animal's wrist movements. The target was an open circle whose inside diameter equaled $8^{\circ}$ of wrist movement. The location of the target on the screen was under computer control. The monkey initiated a trial by placing the cursor in the target that was located in the center of the screen. After a variable "hold" period (0.75 to $1.5 \mathrm{~s})$, a second target appeared at a peripheral location on the screen. When the central hold target was extinguished (1 to $3 \mathrm{~s}$ later), the animal was required to move the cursor to the peripheral target in less than $200 \mathrm{~ms}$. The peripheral target required a $20^{\circ}$ change in the angle of the wrist joint. The monkey received considerable training on this task (over 8 years), and its performance was quite stable. Further details about the task are described in (12).

12. D. S. Hoffman and P. L. Strick, J. Neurophysiol. 73 891 (1995); S. Kakei, D. S. Hoffman, P. L. Strick, Soc. Neurosci. Abstr. 23, 2096 (1997); ibid. 24, 2108 (1998).

13. D. S. Hoffman and P. L. Strick, J. Neurophysiol. 81 319 (1999).

14. All experimental procedures were conducted according to National Institutes of Health guidelines and were approved by the appropriate institutional animal care and use committees.

15. Electromyograms (EMGs) were recorded from 15 forearm, 5 upperarm, and 7 shoulder muscles during the task through pairs of single-stranded stainless- steel wires placed transcutaneously into each muscle. We stimulated through each intramuscular electrode (10 pulses at $50 \mathrm{~Hz}, 10$ to $500 \mu \mathrm{A}$ ) to identify the muscle by observing evoked twitches. The muscles studied included abductor pollicis longus (APL), extensor digitorum 2,3 (ED2,3), extensor digitorum 4,5 (ED4,5), extensor digitorum communis (EDC), flexor digitorum profundus (FDP), flexor digitorum sublimi (FDS), extensor carpi radialis brevis (ECRB), extensor carpi radialis longus (ECRL), extensor carpi ulnaris $(E C U)$, flexor carpi radialis (FCR), flexor carpi ulnaris (FCU), palmaris longus (PL), pronator teres, supinator brachioradialis, brachialis, triceps (long and latera heads), biceps (short and long heads), clavicular deltoid, acromio deltoid, pectoralis major, teres major teres minor, latissimus dorsi, and trapezius. We sampled the same 15 forearm muscles in a second trained monkey and observed comparable patterns of activity.

16. In general, proximal muscles were either silent or did not display phasic task-related activity before movement onset for all three postures. Only acromio deltoid was phasically active before movement onset. However, this activity was present only for one posture. The relative absence of activity in proxima muscles may be due to the fact that the forearm and upper arm were supported and the monkey received extensive training on the task.

17. Wrist: ECRB, ECRL, ECU, and FCR; finger: APL, EDC, and $E D 2,3$. The other finger and wrist muscles that we sampled either were relatively inactive during the task (ED4,5, FCU, FDS, and PL) or displayed activity that began after movement onset (FDP) [see also 13, p. 321]. Supinator and pronator teres were active during the task, but their onset time and directional tuning were irregular.

18. A. B. Schwartz, R. E. Kettner, A. P. Georgopoulos, J. Neurosci. 8, 2913 (1988); for muscles, PD was calculated in a time window of $\pm 25 \mathrm{~ms}$ relative to the movement onset. This time window was determined to include muscle activities for both initiation and peak acceleration of the movement. For neurons, PD was calculated in a time window of -100 to $0 \mathrm{~ms}$ relative to the movement onset in order to exclude the influence of feedback signals.

19. $n=7$ muscles (17), each muscle sampled three to four times (total number of recordings $=23$ ).

20. We used conventional techniques for extracellular recording of single-neuron activity in M1 of one monkey (2). For this purpose, a recording chamber was implanted aseptically under inhalation anesthesia ( $3 \%$ isofluorane $+1 \% \mathrm{~N}_{2} \mathrm{O}$ )

21. In every wrist posture, a data file consisted of 5 to 10 movements for each of eight target locations. Each target location was presented in a randomized block design

22. We sampled the activity of single neurons in a large portion of $\mathrm{M} 1$, including all of the arm representation and adjacent portions of the face and hindlimb representations. The body part represented at each recording site was identified by intracortical stimulation (pulse duration $=0.2 \mathrm{~ms}, 333 \mathrm{~Hz}$ for $60 \mathrm{~ms}$ ) to evoke movements or contractions of muscles. Taskrelated neurons that were directionally tuned were found only in the region of M1 that contained the representation of the wrist and fingers (that is, the hand area of M1). Most task-related neurons were recorded at sites where stimulation evoked contractions of one or a few forearm muscles at thresholds $\leq 15 \mu \mathrm{A}$. We also examined the peripheral receptive fields for recorded neurons. All but three of the task-related neurons in which a receptive field could be identified were activated by passive movements of the wrist or palpation of forearm muscles (or both). The other task-related neurons were activated by passive movements of all the fingers in concert.

23. We used a bootstrapping method to identify whether a neuron was directionally tuned $(P<0.01)$ [D. ] Crammond and J. F. Kalaska, Exp. Brain Res. 108, 45 (1996)]

24. Confidence intervals of PDs of M1 neurons were calculated by using a bootstrapping method [L. E. Sergio and J. F. Kalaska, J. Neurophysiol. 80, 1577 (1998)].

25. The properties of extrinsic- and intrinsic-like neurons had many features in common. The threshold for evoking movement or muscle contraction with intracortical stimulation was 2 to $30 \mu \mathrm{A}$ (mean $\pm \mathrm{SD}=$ $10.7 \pm 6.8 \mu \mathrm{A}, n=44$ ) at the recording sites of extrinsic-like neurons and 5 to $30 \mu \mathrm{A}$ (mean $\pm \mathrm{SD}=$ $12.0 \pm 6.6 \mu \mathrm{A}, n=28$ ) at the sites of muscle-like neurons. The latency for a change in activity related to movement onset was -120 to -70 ms (mean \pm $\mathrm{SD}=-97.0 \pm 15.3 \mathrm{~ms}, n=44$ ) for extrinsic-like neurons and -130 to $-80 \mathrm{~ms}$ (mean $\pm \mathrm{SD}=$ $-93.6 \pm 20.8 \mathrm{~ms}, n=28$ ) for muscle-like neurons. We found no systematic differences in the periphera input that activated extrinsic- and muscle-like neurons. All directions of movement were equally represented in both populations of extrinsic- and musclelike neurons. We also did not find any systematic difference in the amplitude and time course of spikes or spontaneous discharge rate between extrinsic- and muscle-like neurons.

26. Reviewed in J. M. Hollerbach, Trends Neurosci. 5, 189 (1982); J. F. Soechting and M. Flanders, Annu. Rev. Neurosci. 15, 167 (1992).

27. Alexander and colleagues (7) found a relatively small number of neurons in $M 1$ (11/79) that displayed movement-related activity that was "target-dependent," that is, the activity of these neurons was specific to acquiring a particular target, independent of the pattern of muscle activity or the direction of joint movement required. Our behavioral task did not dissociate the direction of action in space from target location. Thus, it is possible that some of the extrinsic-like neurons recorded in our study would be target-dependent neurons. On the other hand, the relative number of extrinsic-like neurons we found (44/ 88 ) is far greater than the small number of targetdependent neurons observed by Alexander and colleagues. This makes it likely that the majority of our extrinsic-like neurons are not target-dependent, but represent a distinct class of neurons that encode direction of action in space. This proposal will need to be tested in future studies that actually perform the appropriate dissociation.

28. We thank A. P. Georgopoulos, S. I. Helms-Tillery, J. F. Kalaska, and L. E. Sergio for consultation on the statistical analyses used in this study. We thank $E$. Stappenbeck for dedicated care and training of the animal; K. Hughes and M. O'Malley-Davis for technical assistance; and M. Page and W. Hartz for development of computer programs. Supported by funds from the Department of Veterans Affairs, Medical Research Service, to P.L.S.

31 March 1999; accepted 26 August 1999 